\title{
AN EASIER ENUMERATION OF SELF-COMPLEMENTARY GRAPHS
}

\author{
by C. R. J. CLAPHAM
}

(Received 6th October 1983)

\section{Introduction}

The number of self-complementary (s.c.) graphs and digraphs with a given number of vertices was found by R. C. Read in [1]. That paper used a special case of De Bruijn's generalisation of Polya's theorem that involved the cycle-index of $G_{n}$, the group of permutations of pairs of vertices induced by permutations of the vertices. We obtain Read's formulae by using only well-known elementary facts about s.c. graphs and their complementing permutations.

\section{Self-complementary graphs with $4 N$ vertices}

Let $\tau$ be a complementing permutation for a s.c. graph $G$ with $4 N$ vertices, that is to say, $\tau$ is a permutation of the vertices that maps $G$ onto its complement $\bar{G}$. It is wellknown that the cycles of $\tau$ have lengths that are multiples of 4. Suppose that $\tau$ consists of $k_{s}$ cycles of length $4 s(s=1,2, \ldots, N)$. For example, if

$$
\tau=\left(v_{1} v_{2} v_{3} v_{4}\right)\left(v_{5} v_{6} v_{7} v_{8}\right)\left(v_{9} v_{10} v_{11} v_{12} v_{13} v_{14} v_{15} v_{16}\right)
$$

then $k_{1}=2, k_{2}=1$ and $k_{s}=0$ for all other values of $s$.

We take vertices $v_{1}, v_{2}, \ldots, v_{4 N}$ and find the number of ways in which edges can be introduced so that the result is an s.c. graph with $\tau$ as a complementing permutation. We have to consider adjacencies (i) between vertices in the same cycle of $\tau$, (ii) between vertices in different cycles of $\tau$ of the same length, and (iii) between vertices in cycles of $\tau$ of different lengths:

(i) The number of adjacencies to be decided between vertices in a cycle of length $4 s$ is equal to $2 s$. For the adjacencies are determined once it has been decided whether the first vertex is to be adjacent or not adjacent to each of the following $2 s$ vertices. For example, in the cycle

$$
\left(v_{9} v_{10} v_{11} v_{12} v_{13} v_{14} v_{15} v_{16}\right)
$$

all the adjacencies are determined once it is known whether or not $v_{9}$ is adjacent to $v_{10}$, $v_{11}, v_{12}$ and $v_{13}$.

(ii) The number of adjacencies to be decided between vertices in different cycles of the same length $4 s$ is equal to $4 s$. For the adjacencies are determined once it has been 
decided whether the first vertex of one cycle is to be adjacent or not adjacent to each of the vertices in the other cycle.

(iii) In a similar way, it is not hard to see that the number of adjacencies to be decided between vertices in a cycle of length $4 \alpha$ and those in a cycle of length $4 \beta$ is equal to $d(4 \alpha, 4 \beta)$, where this denotes the highest common factor of $4 \alpha$ and $4 \beta$.

Since there are $k_{s}$ cycles of length $4 s, \frac{1}{2} k_{s}\left(k_{s}-1\right)$ pairs of cycles of length $4 s$, and $k_{\alpha} k_{\beta}$ pairs of cycles one of length $4 \alpha$ and one of length $4 \beta(\alpha<\beta)$, the total number of adjacencies to be decided is $P$, where

$$
\begin{aligned}
P & =\sum_{s=1}^{N}\left(k_{s} \cdot 2 s+\frac{1}{2} k_{s}\left(k_{s}-1\right) \cdot 4 s\right)+\sum_{1 \leqq \alpha<\beta \leqq N} k_{\alpha} k_{\beta} d(4 \alpha, 4 \beta) \\
& =2 \sum_{s=1}^{N} s k_{s}^{2}+4 \sum_{1 \leqq \alpha<\beta \leqq N} k_{\alpha} k_{\beta} d(\alpha, \beta)
\end{aligned}
$$

(see page 102 of [1]). Then there are $2^{P}$ ways of choosing which adjacencies to introduce and thus the number of labelled s.c. graphs with this $\tau$ as complementing permutation is $2^{P}$.

Now there is an easily obtained result, sometimes known as Cauchy's formula (see, for example, page 123 of [2]), giving the number of elements of a symmetric group which have any particular cycle structure. This gives the number of elements of $S_{4 N}$ with cycle structure consisting of $k_{s}$ cycles of length $4 s(s=1,2, \ldots, N)$ as

$$
\frac{(4 N) !}{\prod_{s=1}^{N}(4 s)^{k_{s}} \cdot k_{s} !}
$$

This then is the number of possible choices for $\tau$ and consequently

$$
\sum_{(k)} \frac{(4 N) !}{\prod(4 s)^{k_{s}} \cdot k_{s} !} 2^{P}
$$

(where the summation $\sum_{(k)}$ is for all sets $k_{1}, k_{2}, \ldots$ such that $\sum s k_{s}=N$ ) gives the number of complementing permutations with all possible labelled s.c. graphs corresponding to each.

Now, for a s.c. graph $G$ with $4 N$ vertices, let $m=|\operatorname{Aut}(G)|$, the order of the automorphism group of $G$. It is elementary that the number of complementing permutations for $G$ is then equal to $m$. Also the number of different labellings of $G$ is equal to $(4 N) ! / m$. So the number of labelled s.c. graphs with all possible complementing permutations corresponding to each is equal to

$$
m \frac{(4 N) !}{m} \sigma_{4 N}
$$

where $\sigma_{4 N}$ is the number of s.c. graphs with $4 N$ vertices. Equating this to the number 
obtained at the end of the previous paragraph, we obtain the formula given in (1):

$$
\sigma_{4 N}=\sum_{(k)} \frac{2^{P}}{\prod(4 s)^{k_{s}} \cdot k_{s} !}
$$

where $P$ is as above.

\section{Self-complementary graphs with $4 N+1$ vertices}

If there are $4 N+1$ vertices, a complementing permutation $\tau^{\prime}$ has the same form as the $\tau$ considered above with an additional cycle of length one. This additional vertex must be adjacent to either the odd-numbered or the even-numbered vertices in any particular cycle of $\tau$, and so the number of additional adjacencies to be decided is just equal to the number of cycles in $\tau$. Consequently, the formula for $\sigma_{4 N+1}$ is just the same as that for $\sigma_{4 N}$, with $P$ replaced by $P^{\prime}$, where $P^{\prime}=P+\sum k_{s}$.

\section{Self-complementary digraphs}

The above method can clearly be copied to find the number $\bar{\sigma}_{2 N}$ of s.c. digraphs with $2 N$ vertices. The result $\bar{\sigma}_{2 N}=\sigma_{4 N}$ is obtained, but the method does not seem to give any new ideas for a natural one-to-one correspondence between the s.c. digraphs with $2 \mathrm{~N}$ vertices and the s.c. graphs with $4 N$ vertices.

\section{REFERENCES}

1. R. C. ReAD, On the number of self-complementary graphs and digraphs, J. London Math. Soc. 38 (1963), 99-104.

2. C. Berge, Principles of Combinatorics (Academic Press, New York and London, 1971).

UNIVERSITY OF ABERDEEN 